\title{
Modeling Driver Behavior at Roundabouts: Results from a Field Study
}

\author{
M. Zhao, D. Käthner, M. Jipp, D. Söffker, and K. Lemmer
}

\begin{abstract}
Advanced Driving Assistance Systems could improve driving safety and comfort by supporting drivers in their driving task. To realize intelligent assistance, driver behavior prediction and recognition is an important challenge. Therefore, the aim of this study is to develop a method to predict whether a vehicle, having entered a roundabout, will choose an upcoming exit or stay within the roundabout. A field study has been conducted to collect driving behavior data for analyzing and modeling human driver behavior in interaction with roundabouts. Support vector machines proved to be a robust and efficient classification method for the roundabout leaving/ staying pattern recognition problem. From the experimental results the vehicles position can be estimated, for which the prediction becomes reliable. The steering wheel angle and angle velocity also proved to be able to provide sufficient information to predict the driver behavior at the investigated roundabouts.
\end{abstract}

\section{INTRODUCTION}

Roundabouts are well known to cause fewer traffic accidents than traditional intersections, which is not the case for bicycles unfortunately [1]. Often, crashes between vehicles and bicycles happen because car drivers can overlook bicycles [2]. One critical situation is demonstrated in Fig. 1: The driver of the red car enters the roundabout and may look to the left to see if there is another car in the roundabout. If, at the same time, a bicycle driver overtakes the car driver on the right side, the car driver is in danger of overlooking the bicycle.

One solution for decreasing the probability of such accidents is to implement warning systems which highlight the danger in case the driver seems to overlook a potential risk [3]. Therefore, these warning systems need to be able to reliably predict the future behavior of the car driver as early as possible. Then, a warning can be issued in time. Thus, the aim of this study is to develop a model which allows predicting the behavior of car drivers when they drive through roundabouts.

M. Zhao is with the Institute of Transportation Systems, German Aerospace Center (DLR), Braunschweig, Germany (e-mail: min.zhao@dlr.de).

D. Käthner is with the Institute of Transportation Systems, German Aerospace Center (DLR), Braunschweig, Germany (e-mail: david.kaethner@dlr.de).

M. Jipp is with the Institute of Transportation Systems, German Aerospace Center (DLR), Braunschweig, Germany (e-mail: meike.jipp@dlr.de).

D. Söffker is with the University of Duisburg-Essen, Chair of Dynamics and Control, Duisburg, Germany (e-mail: soeffker@uni-due.de).

K. Lemmer is with the Institute of Transportation Systems, German Aerospace Center (DLR), Braunschweig, Germany (e-mail: karsten.lemmer@dlr.de).

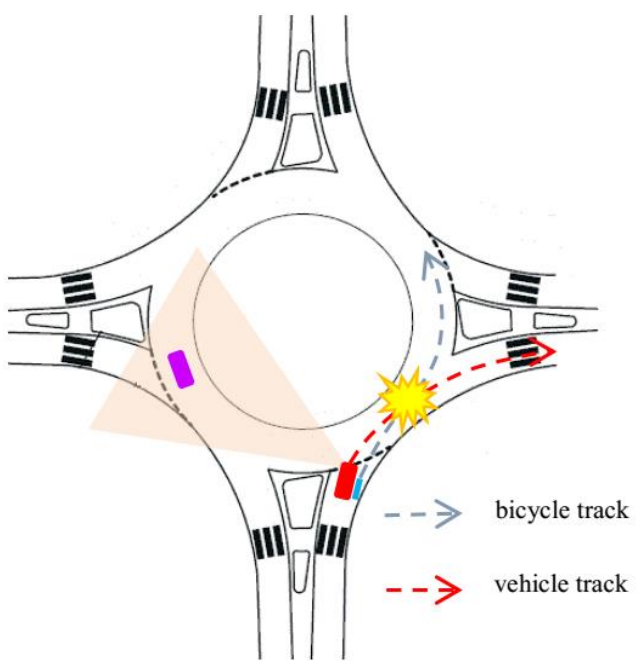

Fig. 1. Dangerous situation at roundabout.

\section{A. Motivation}

Roundabouts have gained importance and are considered essential components of road infrastructure [4]. For instance, there are already around 4,800 modern roundabouts in the US until December 2015 [5]. A study shows that converting intersections to roundabouts reduces injury crashes by $81 \%$ in the US [6]. The accidents that still occur at roundabouts are due to (a) drivers who indicate their intention of leaving or staying in the roundabout incorrectly, and (b) drivers who incorrectly predict the behavior of other road users [6].

One way to prevent or mitigate crashes between cyclists and cars is to establish in-car warning systems warning their car drivers in case of overlooking potential risks. These systems can work efficiently, if they are enabled to predict the upcoming behavior of their drivers and implement an appropriate strategy [7].

\section{B. State of the Art}

Many researchers focused on scenarios on the motor way to predict if driver will follow heading car or overtake it [8-15]. As input variables, information about driving behavior such as steering angle, speed, lateral position, acceleration, and time to collision [8, 9] and about gaze behavior [10] was used successfully. For modeling, Pentland (1999), Kuge (2000), and Mizushima (2006) drew on Hidden Markov Models (HMMs). The researchers assumed that human intention is a sequence of internal mental states that cannot be observed but modeled by abstracting the observable behavior [11-13]. Tango (2009) compared three machine learning techniques for modeling: Neural Network (NN), Hidden Markov Model (HMM), and Support Vector Machine (SVM). As conclusions it was stated that $\mathrm{NN}$ and SVM have 
comparable performances on two-pattern recognition (car-following/ lane-changing) with 99\% detection rate; HMM achieve $97 \%$ detection rate on three-pattern recognition: car following, lane changing, and lane keeping on free lane [9].

Researchers also focused on predicting car turning behavior at (urban) intersections [16-20]. Naito (2008) used vehicle control signals to develop a prediction model based on K-means clustering which can reach the detection rate $95.6 \%$ at 5 seconds before the intersections [16]; Lefèvre (2011) proposed a Bayesian network, which combined observations from the vehicle behavior and information about the geometrical characteristics of the intersection [17]. Aoude (2011) validated SVM and HMM using naturalistic driving data at intersection [18]. Gadepally (2014) predicted driver turning behavior at intersections with HMM, based on vehicle dynamics information [19].

Predicting human car driving behavior at roundabouts has hardly been in the focus of studies. Mudgal (2014) modelled speed profiles at roundabouts using a Bayesian inference methodology and simulated circulating speed and maximum accelerations [21]. Sun (2016) assessed driving behavior at roundabout based on the visual-motor coordination of individual drivers [22]. However, a method for inferring driver behavior at roundabouts and especially of whether or not a driver will exit the roundabout at the next exit is not yet available. Therefore, this study is focused on developing a model that predicts driver behavior when drivers approach an exit of a roundabout.

\section{Research Questions}

The main research question is how to model whether a car driver continues driving in a roundabout (going straight) or whether a car driver leaves the roundabout at the next exit (turning right, see Fig. 2):

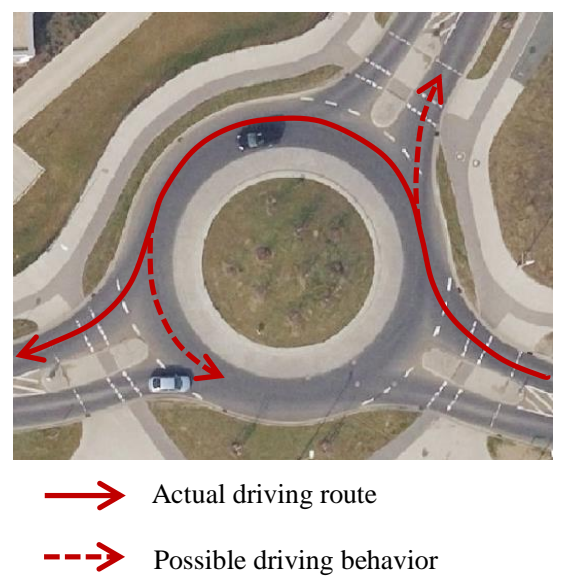

Fig. 2. Possible driver behavior in roundabouts.

\section{METHOD OF MODELING DRIVER BEHAVIOR}

To predict the leaving/staying behavior of drivers at roundabouts, the data which represent two different driver behavior categories (leaving and staying) were acquired while driving through roundabouts. By learning the characteristics of these data, a prediction model is developed. With this model, new driving data whose category membership is unknown can be identified which category they belong to. When the category of the driving data is determined, the leaving/staying behavior will be predicted. Therefore, this is a classification problem, and the model can be trained with machine learning algorithms.

\section{A. Data Acquisition}

Seven participants were asked to drive through a track at least 30 times in the city of Braunschweig (Germany). This track included three roundabouts with different geometrical layouts and required the participants to take each pair of entry and exit for the three roundabouts. The participants used a specific car equipped with powertrain, steering angle sensor, odometer, GPS receiver. Herewith, driving behavior (steering angle, steering angle velocity, acceleration, velocity, and position) was captured for each participant. The logging frequency is $50 \mathrm{~Hz}$. TABLE I and II show some example data of the driving behavior for two driving patterns: leaving roundabouts and staying at roundabouts.

TABLE I. Example data for driving pattern of leaving roundabouts.

\begin{tabular}{|c|c|c|c|}
\hline $\begin{array}{c}\text { Coordinated } \\
\text { Universal Time } \\
(\mathbf{m s})\end{array}$ & $\begin{array}{c}\text { Velocity } \\
(\mathbf{k m} / \mathbf{h})\end{array}$ & $\begin{array}{c}\text { Acceleration } \\
\left(\mathbf{m} / \mathbf{s}^{2}\right)\end{array}$ & $\begin{array}{c}\text { Steering } \\
\text { angle velocity } \\
(\mathbf{g r a d} / \mathbf{s})\end{array}$ \\
\hline 1427457433553 & 22.0000 & 0.5775 & -3771 \\
\hline 1427457433573 & 21.9300 & 0.2250 & -3771 \\
\hline 1427457433593 & 21.8100 & 0.2050 & -4114 \\
\hline 1427457433613 & 21.8700 & 0.4775 & -4457 \\
\hline 1427457433633 & 21.8700 & 0.6500 & -4114 \\
\hline $\begin{array}{c}\text { Coordinated } \\
\text { Universal Time } \\
(\mathbf{m s})\end{array}$ & $\begin{array}{c}\text { Steering } \\
\text { angle } \\
(\mathbf{g r a d})\end{array}$ & Longitude & Latitude \\
\hline 1427457433553 & 147.0000 & 0.18390771 & 0.91305843 \\
\hline 1427457433573 & 143.9812 & 0.18390770 & 0.91305844 \\
\hline 1427457433593 & 139.5188 & 0.18390768 & 0.91305845 \\
\hline 1427457433613 & 135.0125 & 0.18390766 & 0.91305847 \\
\hline 1427457433633 & 131.9937 & 0.18390764 & 0.91305848 \\
\hline
\end{tabular}

TABLE II. Example data for driving pattern of staying at roundabouts.

\begin{tabular}{|c|c|c|c|}
\hline $\begin{array}{c}\text { Coordinated } \\
\text { Universal Time } \\
(\mathbf{m s})\end{array}$ & $\begin{array}{c}\text { Velocity } \\
(\mathbf{k m} / \mathbf{h})\end{array}$ & $\begin{array}{c}\text { Acceleration } \\
\left(\mathbf{m} / \mathbf{s}^{2}\right)\end{array}$ & $\begin{array}{c}\text { Steering } \\
\text { angle velocity } \\
(\mathbf{g r a d} / \mathbf{s})\end{array}$ \\
\hline 1427456301773 & 18.8700 & 1.5525 & -1029 \\
\hline 1427456301793 & 18.8100 & 1.2250 & -686 \\
\hline 1427456301813 & 18.9300 & 0.9050 & -1029 \\
\hline 1427456301833 & 19.1200 & 0.7975 & -1029 \\
\hline 1427456301853 & 19.2500 & 0.9050 & -686 \\
\hline $\begin{array}{c}\text { Coordinated } \\
\text { Universal Time } \\
(\mathbf{m s})\end{array}$ & $\begin{array}{c}\text { Steering } \\
\text { angle } \\
(\mathbf{g r a d})\end{array}$ & Longitude & Latitude \\
\hline 1427456301773 & 183.0062 & 0.1839078 & 0.9130585 \\
\hline 1427456301793 & 183.0062 & 0.1839078 & 0.9130585 \\
\hline 1427456301813 & 181.5188 & 0.1839078 & 0.9130585 \\
\hline 1427456301833 & 179.9875 & 0.1839078 & 0.9130586 \\
\hline 1427456301853 & 179.9875 & 0.1839078 & 0.9130586 \\
\hline
\end{tabular}




\section{B. Data Pre-processing}

The data relevant for modeling, which are the data between adjacent exits of the roundabouts, had to be selected. Therefore, the center of each roundabout and the entry corners of the roundabouts were calculated (see the red dots in Fig. 3b and $3 \mathrm{c}$ ) and the data outside of these boundaries were removed (see Fig. 3d and 3e). Then, erroneous data, i.e., wrong position and directions were removed.
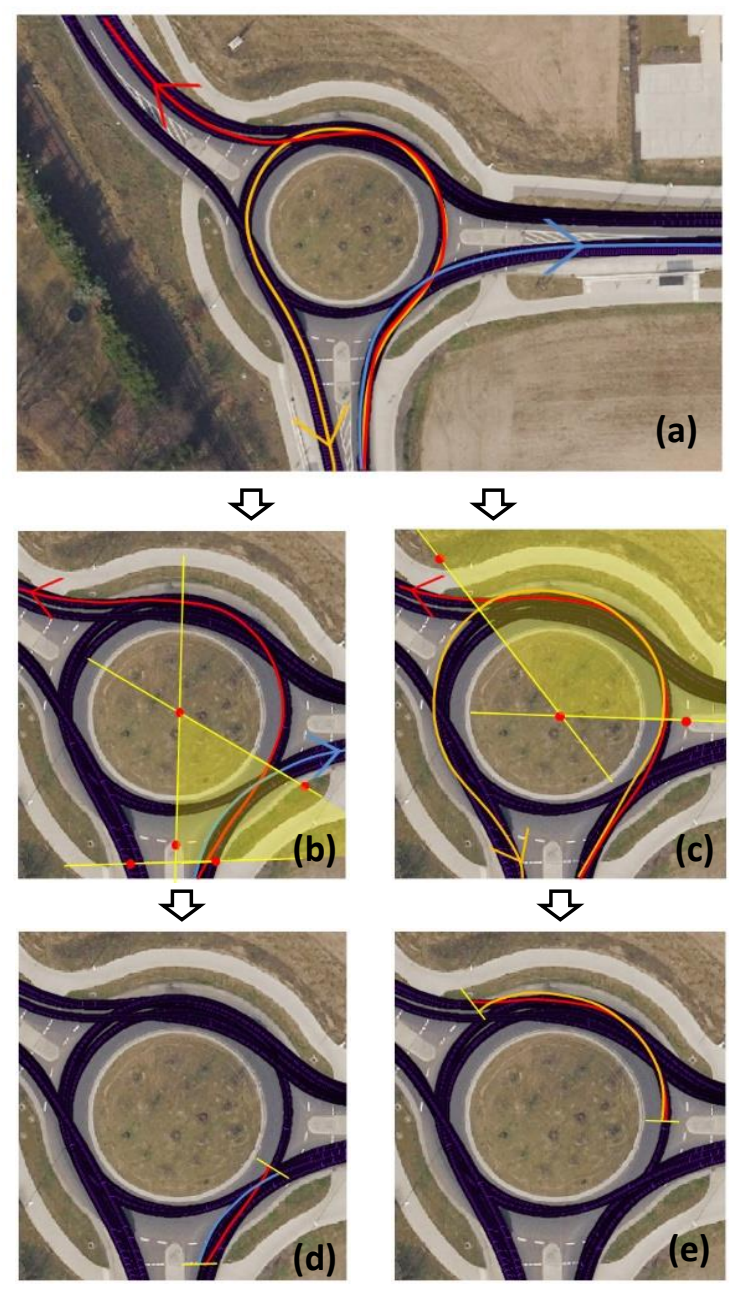

Fig. 3. Selection of relevant data

\section{Driver Behavior Information and Scenario Analysis}

In this study, steering angle and steering angle velocity were selected for leaving/staying behavior prediction at roundabout because they depend on the driving direction. Velocity and acceleration are not suitable features for modeling because they are affected by surrounding traffic and the speed limit.

The steering wheel has different status in different scenarios where the roundabout geometric design and the driving exit are different. To improve the predicting ability of the steering angle and the steering angle velocity, three scenarios were distinguished in this study on the basis of the relationship between the geometry of the roundabouts and the steering wheel status:

- In scenario 1, drivers tend to keep steering to the right when they drive through the roundabout. This is the case if the angle between the entry and exit is less than $110^{\circ}$ and if the entry and exist are adjacent to each other (see Fig. 4).

- In scenario 2, drivers tend to steer to the right first to enter the roundabout but then steer to the left to follow it and last turn to the right to leave it. This is the case if the angle between the entry and exit is more than $110^{\circ}$ and if the entry and exit are adjacent to each other (see Fig. 5).

- In scenario 3, drivers tend to steer to the left to follow the roundabout, and steer to the right to exit the roundabout. This is the case if the angle between the entry and exit is more than $110^{\circ}$ and if the entry and exit are not adjacent to each other. In contrast to scenario 2, the drivers do not steer to the right at the beginning of prediction, because they are already in the roundabout (see Fig. 6).

Three sub-models have been developed to predict the driving behavior in these three scenarios. Sub-model 1, sub-model 2, and sub-model 3 correspond to scenario 1, scenario 2 , and scenario 3 respectively. When the driver is entering a roundabout, the sub-model 1 or sub-model 2 (the choice depends on the angle between the entry and the adjacent exit) are used to predict whether the driver would take the next exit to leave the roundabout. If the answer is no, then the sub-model 3 is used to predict whether the driver would leave the roundabout through next exits.

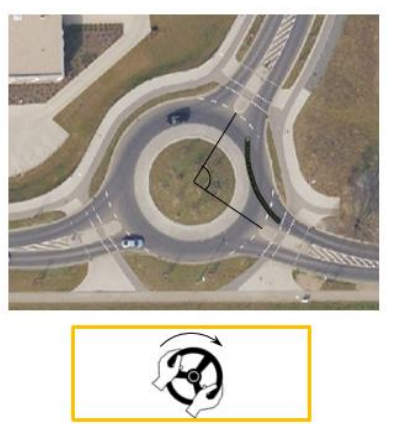

Fig. 4. Scenario 1
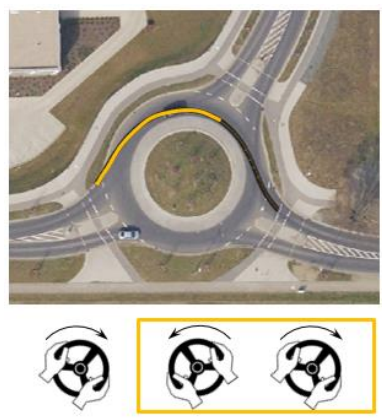

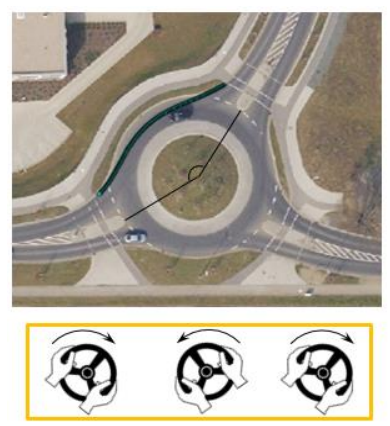

Fig. 5. Scenario 2

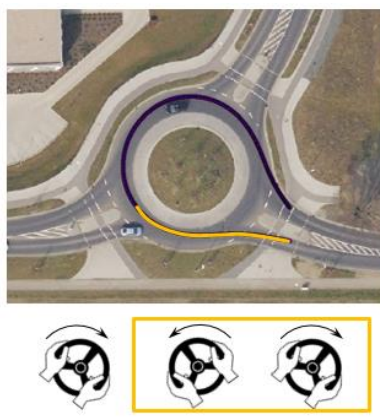

Fig. 6. Scenario 3

\section{Classification Using Support Vector Machine}

The leaving/staying behavior prediction is a binary classification problem. SVM has been proven to be an 
effective and robust method for binary classification problems [23]. So, in this study SVM was adopted.

The modeling process is divided into four steps:

1. Feature extraction: To yield related features for modeling, the data of steering angle and steering angle velocity which is on the section between entry and exit for each scenario and each participant were mapped from the time line to the distance line with interpolation. This mapping procedure was chosen because each driver drives through roundabouts with different speeds. For interpolation, the distance lines were scaled from the origin at the entry line. The drives were divided into 10 parts evenly by 11 points, and these points were defined as "recognition sites" (red dots in Fig. 7). At each recognition site, the steering angle value and the steering angle velocity value were extracted as the features for the classification.

2. Scaling features: The features were scaled to the range $[-1,1]$ to avoid variables in differing ranges dominating those in smaller ranges [24].

3. Splitting the data set: 309 drive samples for scenario 1 , 302 for scenario 2, and 450 for scenario 3 are used. For each scenario, the data set was split into training data set $(80 \%)$ and testing data set $(20 \%)$.

4. Applying cross validation: 5-fold cross-validation was used to the training data set to identify the best parameters for the SVM model, and the model was validated with the testing data set.

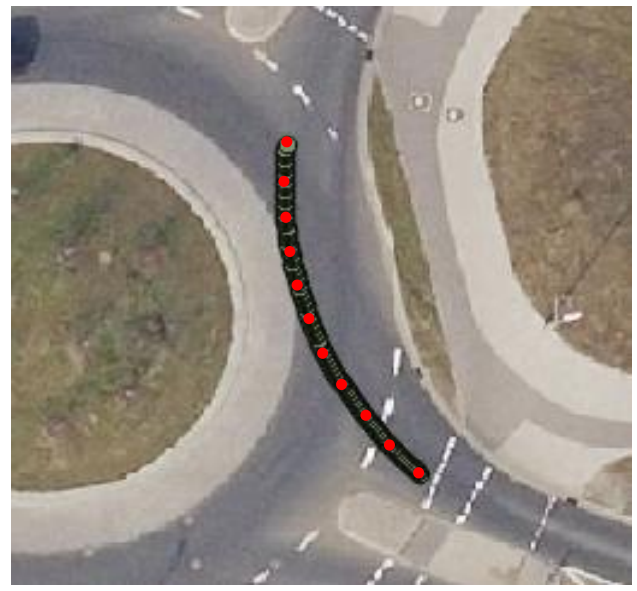

Fig. 7. Recognition sites on one drive

At each recognition site for each scenario, these four-step modeling process was applied. To measure the performances of the models, the recognition accuracy is defined as the ratio between the number of instances correctly classified and the number of instances presented in the test dataset. The recognition accuracy at each recognition site was calculated. The distance from each recognition site to the oncoming exit is also calculated, so the related position of the recognition site is known. Given threshold of 95\%, the recognition sites and their distances to the exit were identified, where the recognition accuracy was the earliest above $95 \%$.

\section{RESULTS}

In this study, linear and radial basis function kernels were tested with different parameters for the classification. The linear kernel with $\mathrm{C}=100$ performed best. The classification process at each site needed $0.01 \mathrm{~s}$.

The results are visualized in Figures 8, 9, and 10 for three scenarios:

- Scenario 1: The recognition accuracy reached an accuracy of $98.1 \%$ at recognition site 4 with a distance of $13.8 \mathrm{~m}$ before the exit (see Fig. 8).

- Scenario 2: The recognition accuracy reached an accuracy of $97.4 \%$ at recognition site 7 , which was $11.4 \mathrm{~m}$ away from the exit of the roundabout (see Fig. 9).

- Scenario 3: The recognition accuracy reached $98.5 \%$ at recognition site 6 , which was $14.1 \mathrm{~m}$ away from the exit of the roundabout (see Fig. 10).

\section{CONCLUSION}

The main result obtained is that future human behavior can be predicted at roundabouts. To gain this result, a study was conducted during which data on human behavior at roundabouts was captured in real traffic. A part of the data on steering angle and steering velocity was used as inputs to train a model based on SVM. The resulting model was validated with a test data set. The recognition accuracy is promising: The upcoming behavior could be predicted with a high level of accuracy (larger than 95\%) at a distance of approximately 11 $\mathrm{m}$ before the exit. The results show that the steering angle and the steering angle velocity provide important information for driving behavior prediction at roundabout. The recognition accuracy is getting higher as the recognition site is getting closer to the oncoming exit.

This study has developed the model to predict driving behavior at roundabouts, which fills a research gap of driver behavior modeling at roundabouts. Future work should 1) consider additional behavior variables as feature inputs (such as speed and acceleration) and other classifiers (such as Hidden Markov Model), 2) improve the model for behavior prediction at generic roundabouts, and 3) focus on the required timing for warning assistance systems. 


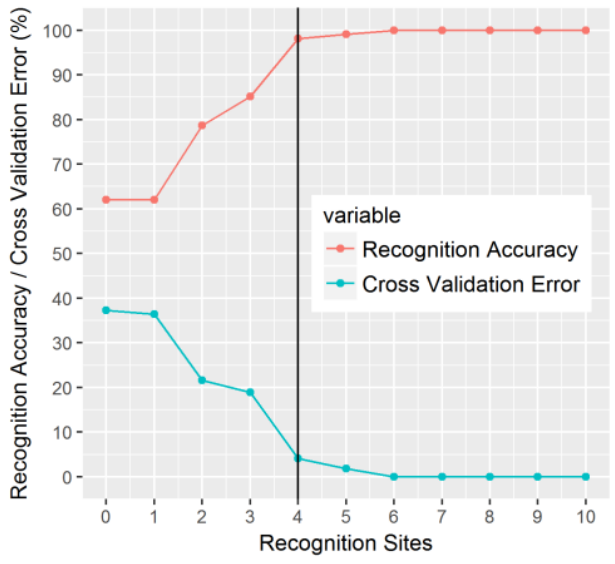

(a)

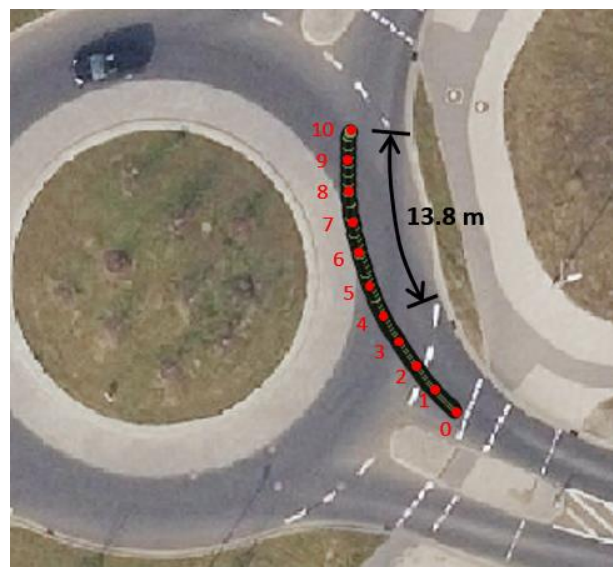

(b)

Fig. 8. Prediction results for scenario 1

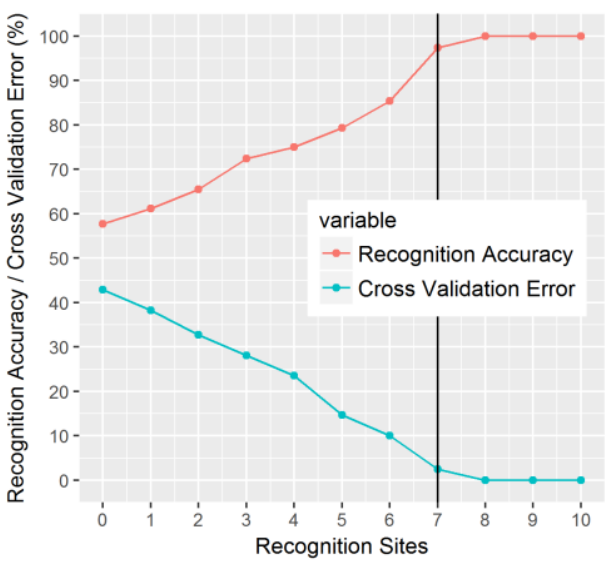

(a)

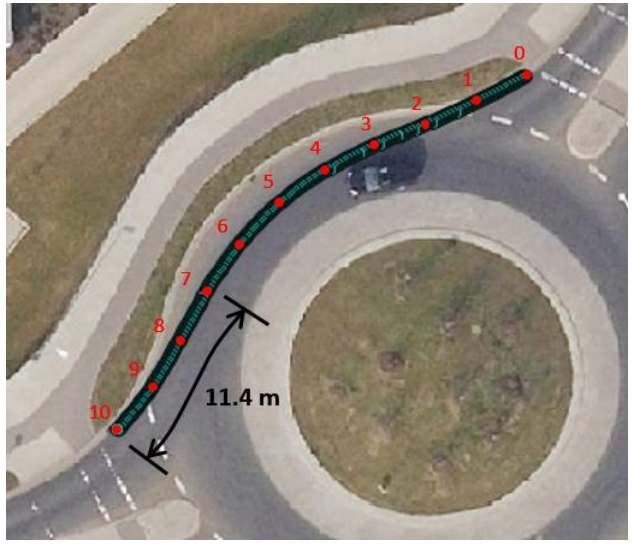

(b)

Fig. 9. Prediction results for scenario 2

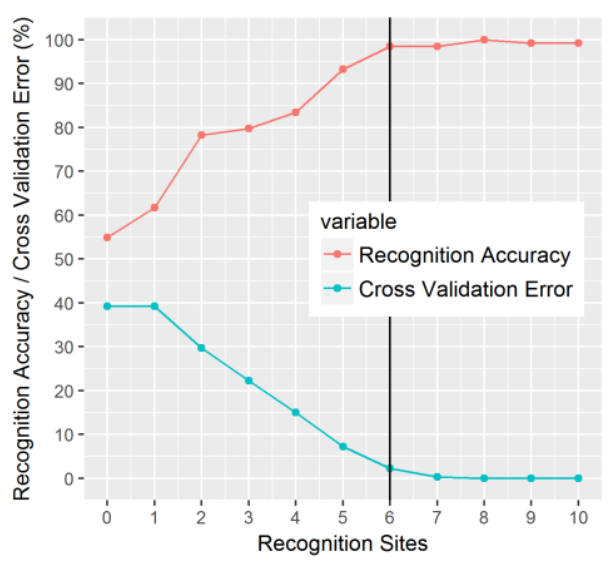

(a)

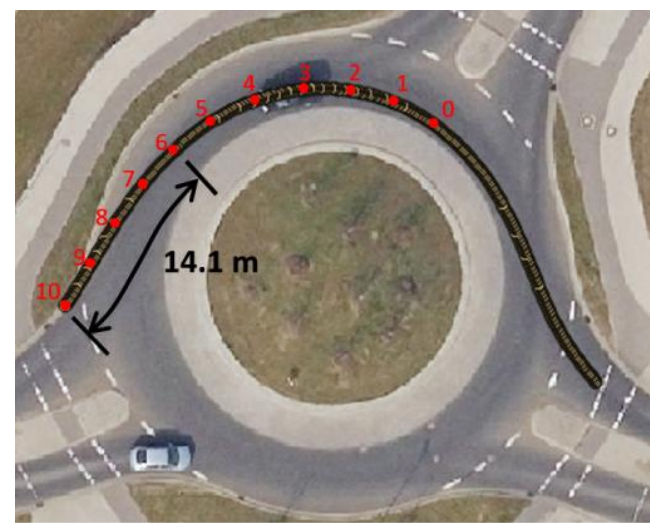

(b)

Fig. 10. Prediction results for scenario 3 


\section{REFERENCES}

[1] T. Hels and I. Orozova-Bekkevold, "The effect of roundabout design features on cyclist accident rate", Accident Analysis \& Prevention, vol. 39(2), pp.300-307, 2007.

[2] M.B. Herslund and N.O. Jørgensen, "Looked-but-failed-to-see-errors in traffic", Accident Analysis \& Prevention, vol. 35(6), pp.885-891, 2003.

[3] J. Werneke and M. Vollrath, " How to present collision warnings at intersections? - A comparison of different approaches", Accident analysis \& prevention, vol. 52, pp.91-99, 2013.

[4] W. Brilon, "Roundabouts: A state of the art in Germany", in National Roundabout Conference, Vail, Colorado, 2005, vol. 16.

[5] B. Baranowski, "History of the modern roundabout." Internet: http://www.roundaboutsusa.com/history.html, [2016].

[6] A. Montella, "Identifying crash contributory factors at urban roundabouts and using association rules to explore their relationships to different crash types", Accident Analysis \& Prevention, vol. 43(4), pp.1451-1463, 2011.

[7] L. He, C.F. Zong, \& C. Wang, "Driving intention recognition and behavior prediction based on a double-layer hidden Markov model", Journal of Zhejiang University SCIENCE C, vol. 13(3), pp.208-217, 2012.

[8] A. Liu and A. Pentland, "Towards real-time recognition of driver intentions", IEEE Conference on Intelligent Transportation System, 1997, pp. 236-241

[9] F. Tango and M. Botta, "ML techniques for the classification of car-following maneuver", In AI* IA 2009: Emergent Perspectives in Artificial Intelligence, Springer Berlin Heidelberg, pp.395-404, 2009.

[10] F. Lethaus, M.R. Baumann, F. Köster, and K. Lemmer, "A comparison of selected simple supervised learning algorithms to predict driver intent based on gaze data", Neural computation, vol. 121, pp.108-130, 2013.

[11] A. Pentland and A. Liu, "Modeling and prediction of human behavior", Neural computation, vol. 11(1), pp.229-242, 1999.

[12] N. Kuge, T. Yamamura, O. Shimoyama, and A. Liu, "A driver behavior recognition method based on a driver model framework", $S A E$ Technical Paper, No. 2000-01-0349, 2000.

[13] T. Mizushima, P. Raksincharoensak, and M. Nagai, "Direct yaw-moment control adapted to driver behavior recognition", in SICE-ICASE, 2006. International Joint Conference, pp. 534-539.

[14] H.M. Mandalia and M.D.D. Salvucci, "Using Support Vector Machines for lane-change detection", In Proceedings of the Human Factors and Ergonomics Society Annual Meeting, 2005, Vol. 49, No. 22, pp. 1965-1969.

[15] S. Bengtsson, "Detection and prediction of lane-changes: A study to infer driver intent using support vector machine", M.S. thesis, Dept. Machine Design, KTH Royal Institute of Technology, Stockholm, Sweden, 2012.

[16] T. Naito, H. Morimoto, T. Ito, T. Yamamoto, and H. Fukumoto, "Driver turning behavior prediction at intersections: improving prediction performance with driver behavior adaptation", Review of automotive engineering, vol. 29(2), pp.157-163, 2008.

[17] S. Lefèvre, "Modeling dynamic scenes at unsignalised road intersections", HAL - Inria, Id: inria-00588758, 2011.

[18] G.S. Aoude, V.R. Desaraju, L.H. Stephens, and J.P. How, "Behavior classification algorithms at intersections and validation using naturalistic data", In Intelligent Vehicles Symposium (IV), IEEE, 2011, pp. 601-606.

[19] V. Gadepally, A. Krishnamurthy, and U. Ozguner, "A framework for estimating driver decisions near intersections", IEEE Transactions on Intelligent Transportation Systems, vol. 15(2), pp.637-646, 2014.

[20] M. Liebner, F. Klanner, M. Baumann, C. Ruhhammer, and C. Stiller, "Velocity-based driver intent inference at urban intersections in the presence of preceding vehicles", Intelligent Transportation Systems Magazine, IEEE, vol. 5(2), pp.10-21, 2013

[21] A. Mudgal, S. Hallmark, A. Carriquiry, and K. Gkritza, "Driving behavior at a roundabout: A hierarchical Bayesian regression analysis", Transportation research part D: transport and environment, vol. 26, pp.20-26, 2014.

[22] Q.C. Sun, J.C. Xia, T. Falkmer, and H. Lee, "Investigating the spatial pattern of older drivers' eye fixation behaviour and associations with their visual capacity". Journal of Eye Movement Research, vol. 9(6), pp.1-16, 2016.

[23] K. Hornik, D. Meyer, and A. Karatzoglou, "Support Vector Machines in R", Journal of statistical software, vol. 15(9), pp.1-28, 2006.

[24] C.W. Hsu, C.C. Chang, and C.J. Lin, "A practical guide to support vector classification". Department of Computer Science, National Taiwan University, Tech. rep., 2003. 